\title{
Bioética y género: análisis desde la vulnerabilidad humana
}

\section{Bioethics and gender: analysis from human vulnerability}

\author{
Ma. Elizabeth de los Ríos Uriarte*
}

\section{Resumen}

El artículo aborda la relación entre la bioética y la ideología de género a partir de la noción de vulnerabilidad. Así, se afirma que la segunda pone a la persona en una situación de mayor vulnerabilidad al carecer de un fundamento ontológico que la sostenga y, por ende, pudieran desprenderse, de esto, prácticas poco éticas para con quienes se engloban dentro de alguno de los grupos residuales de la misma. Se parte, por tanto, del origen de la ideología de género para, posteriormente, ubicar las problemáticas más frecuentes que se presentan, en el terreno de la Bioética, a partir de la vulnerabilidad antropológica y social. Por último se rescata el papel de la Bioética en la intervención de las poblaciones vulnerables y vulneradas en el marco del tema del género.

Palabras clave: género, bioética, vulnerabilidad, ideología, persona.

\footnotetext{
* Doctora en Filosofía por la Universidad Iberoamericana. Profesora titular de la Cátedra de Bioética Clínica de la Facultad de Bioética de la Universidad Anáhuac México. Correo electrónico: marieli829@hotmail.com

Recibido el 04 de septiembre de 2019. Entregado el 30 de septiembre de 2019.
}

Medicina y Ética - Octubre-Diciembre 2019 - Vol. 30 - Núm. 4 


\section{La separación entre sexo y género: el surgimiento de los estudios de género}

¿Qué es lo constitutivamente humano, la naturaleza o la cultura? En otras palabras, ¿el sexo entendido como lo dado por la naturaleza humana y, por ende, fijo e inamovible, o el género que es producto de la libertad y creatividad humanas que conforma el ámbito de lo cultural, por ende, cambiante y poroso? Ésta parece ser la pregunta que denota diversas respuestas que van desde las posturas irreconciliables hasta algunas más centristas; en cualquiera de ella, el tema pasa, necesariamente, por las categorías de «sexo», "género» y las relaciones intrínsecas que éstas establecen en el campo del saber y del poder.

Estas categorías y sus relaciones derivadas constituyen objeto de serios estudios y de profundas reflexiones en nuestro tiempo; así, surgen la inquietud por los estudios de género, la apuesta por la liberación de las mujeres y de las formas consideradas subversivas de sexualidad; es decir, los gays, las lesbianas, los bisexuales, transexuales y los transgénero.

Desde la obra de Simone de Beauvoir El segundo sexo, donde se afirma que «no se nace mujer, se hace mujer» (Beauvoir 2005, 109), hasta los estudios de Michel Foucault en su Historia de la sexualidad en 1979, el debate subyacente entre las dos categorías ha dejado ver el juego de poder que se ejerce bajo las argumentaciones naturalistas en donde se da por hecho y válido que a cada naturaleza corresponden ciertas características y que, por definición las de la mujer han sido las más «débiles e incompletas»; luego entonces, el lugar que ésta ha ocupado en la relación con los varones ha sido siempre inferior por pertenecer al «sexo débil», quedando sujeta a un dominio y control por parte del hombre y reducida a las funciones que le son «inherentes» según su sexo.

De lo anterior se deduce que el estudio de estas dos categorías en ambos autores haya apuntalado el despertar, por un lado, de las mujeres consideradas históricamente inferiores y, por otro lado, un 
radical cuestionamiento de la indisolubilidad entre lo naturalmente dado y lo socialmente construido. Así, surge la idea de entender que si bien puede o no ${ }^{1}$ haber una base biológica para la determinación como mujer o como varón, ésta se encuentra separada de la conducta, de las prácticas, de los modos de ser y de las acciones que la persona pueda ejercer; a éstos se les denominó «género» y se estableció entonces que éste depende de los constructos sociales y que, por ende, es modificable y variable.

Así entonces «sexo» y «género» significaron dos cosas distintas -si no es que opuestas-. El sexo proviene de la naturaleza que, a su vez, representa el ámbito del determinismo por ser fijo e inamovible y sólo pudiendo ser, en la persona, o bien femenino o bien masculino, estableciendo una radical diferencia entre ambos que constituye la episteme precultural que determina y permea las relaciones sociales y justifica las relaciones de poder entre los dos sexos mencionados. Por su parte, el género será el residuo de constructos sociales, de ámbitos culturales que pueden ser variados y heterogéneos; así que el abanico de posibilidades ya no es únicamente binario sino múltiple: de lo femenino o lo masculino se pasa a la relación entre sexos admitiendo la homosexualidad, heterosexualidad, bisexualidad, transexualidad, etcétera.

El género ya no es entonces el ámbito del determinismo si no de la libertad y de la creatividad, en donde ya no depende la conducta de lo que la naturaleza marca, sino de los modos en que se van configurando las relaciones sociales, las leyes, las costumbres, y que van permeando en distintos foros y espacios públicos. El género es la relación y el poder que se ejerce entre y por medio de los cuerpos, son las relaciones asimétricas que encuentra su fundamento en los prejuicios y las construcciones sociales y, por ello, carecen de solidez y de fuerza.

El género se entiende entonces como: «los roles socialmente construidos, comportamientos, actividades y atributos que una sociedad considera como apropiados para hombres y mujeres» (WHO, 2019). 
Algunos ejemplos que ilustran la idea de género como rol social son, por ejemplo, pensar en la figura del varón como el proveedor de cuidados y de sustento, el que necesariamente tiene la última palabra, el que debe darle órdenes a la mujer, el que debe salir a trabajar, el que debe obtener los puestos de autoridad en la vida profesional, el que debe ser físicamente más fuerte y dedicarse a los trabajos considerados «pesados», mientras que la mujer debe ser la que cuide el hogar, a los hijos, la que no debe trabajar, la que debe dedicarse a cocinar y limpiar, la que no puede ocupar puestos de autoridad y debe tener un salario inferior al del varón, la que representa el lado de los sentimientos y las emociones y no el racional y/o lógico, etcétera.

Si bien hay que reconocer que los primeros estudios de género dieron origen a las primeras generaciones de feministas (Bonder, 1998) y su base radicaba en la reivindicación de los derechos que le habían sido negados a la mujer sólo por el hecho de ser mujer, esta crítica provocó ideas muy favorecedoras para el entendimiento de la naturaleza humana como principio de máximo respeto y promoción de la persona. La discusión no sólo hizo aparecer públicamente la vergüenza de actuar conforme a usos y costumbres sin velar por la integridad de la dignidad humana, sino que situó históricamente el hecho de la vulnerabilidad social que margina y excluye sin fundamentos, por ello su voz representó un hito imposible de seguir silenciándose.

Con ello hay que diferenciar entre los estudios de género que se dan a la tarea de analizar y estudiar el hecho de las relaciones entre los sexos de forma objetiva y metódica, y la ideología de género que se deriva de la defensa de la igualdad entre sexos, una vez percibida la desigualdad histórica y defiende la libre elección del género desarraigado del sexo y la hoy llamada perspectiva de género que intenta, bajo prácticas académicas, políticas, institucionales, paliar la desigualdad existente entre el sexo femenino y el sexo masculino, con el objetivo de hacer valer por igual la dignidad de ambos en cuanto personas. En palabras de Burgos: «Entiendo 
como ideología de género la perspectiva teórica de corte radical que concibe la sexualidad como una elección personal sobre un fondo biológico» (Burgos, 2011).

De igual manera, resulta pertinente aclarar algunas nociones que redundan en este estudio y que han sido promovidas a raíz de la separación entre sexo y género. Si se ha aclarado ya con anterioridad que el sexo será lo dado por la combinación cromosómica resultante de los 23 cromosomas de los gametos femeninos y los 23 cromosomas de los gametos masculinos pudiendo resultar en un «XX» como femenino (mujer) y un «XY» como masculino (varón), y admitiendo las muchas variables de las que es susceptible el feto a lo largo de su desarrollo y, por ende, considerando también las cargas hormonales a las que están sujetos (sexo cromosómico y sexo fenotípico) y, por otra parte, el género entendido como los roles y las conductas que se son esperadas tanto para uno como para el otro, entonces podemos diferenciar en primer lugar la identidad sexual como aquella identificación entre el cuerpo de la persona y su psique que le genera emociones y sentimientos de autopertenencia e identificación y reconocimiento de sí mismo y, por ende, de concordancia entre lo que se siente que es y lo que es en realidad.

En la noción anterior se pueden encontrar problemas de identificación de hombres que tienen cuerpo de hombres, carga hormonal de varones pero que se «sienten» mujeres o viceversa.

\subsection{Orientación e identidad sexual: el residuo del liberalismo}

Por su parte, la noción de orientación sexual se refiere a la atracción que siente una persona por otra, ya sea de su mismo sexo o de uno diferente; así, puede haber una persona que sea mujer, es decir, que tenga carga hormonal femenina y física y biológicamente órganos reproductores femeninos, pero que sienta atracción hacia otra mujer o bien hacia el sexo opuesto. 
Ambas definiciones, identidad sexual y orientación sexual, vienen dadas por la separación de las categorías de «sexo» y "género», y son el residuo de movimientos sociales que afirman una «libertad» para elegir lo que desean ser. Este movimiento viene, a su vez, por una exaltación de la autonomía desde el liberalismo.

El liberalismo, cuya tendencia se puede ubicar desde Locke hasta Nozick, enuncia un estado de mínima intervención por parte del estado en la vida de los ciudadanos, defendiendo, a ultranza, tanto la autonomía como la propiedad privada.

La concepción de la autonomía, a diferencia de Kant, es una de corte absolutista en donde ésta se construye con base en las elecciones y valores internos de cada persona con independencia de influencias externas, tradiciones, costumbres, valores sociales $\mathrm{O}$, incluso, leyes y regulaciones. Así, con base en la decisión libre y autónoma cada persona va construyendo su identidad y narrando su vida.

En este orden de ideas, el estado sólo debe fungir como regulador de las conductas sociales mediante una garantía de justa distribución de recursos y oportunidades, pero sin dirigir o prohibir conductas o ámbitos de comportamiento en los individuos.

Uno de los problemas fundamentales del liberalismo en lo tocante a las áreas de la Bioética es el hecho de que las decisiones, al tomarse de forma independiente del contexto y, más aún, de marcos de referencia sociales, culturales o familiares, carecen de fundamentación y continuidad; es decir, son volátiles y, por ende, cambiantes, ya que la circunstancia de vida de cada persona varía según el momento histórico y el tiempo individual. Las emociones que de éste se desprenden son modificables según el entorno; así, se corre el grave riesgo de decidir algo que luego se conciba como equivocado, pero el residuo de nuestras acciones en función de la libertad, es decir, la responsabilidad, no desaparece, no cambia ni se esfuma con el cambio de decisiones; luego entonces queda como remanente incómodo cuando se han tomado decisiones sin una base continuada de valores y preferencias. 
Por otro lado, un segundo problema que representa el liberalismo es que concibe la libertad de la persona de forma absoluta y esto, antropológicamente, es imposible. Nos hallamos, indefectiblemente, arraigados a un cuerpo y en un mundo; por nuestra corporeidad somos frágiles y es nuestro cuerpo y nuestra biología las que marcan muchos modos de vivir y de comportarnos. Además, el cuerpo social, la comunidad en la que quedamos insertos, incluso sin nuestro consentimiento en un primer momento, marca y delimita identidades y formas de pensar y ser; por ende, aun cuando se suponga que el ser humano se puede librar de todas estas estructuras, sigue quedando inserto en un entorno y en un cuerpo que son límite y marcaje de su vida.

Deslindarnos de todo lo que ejerce influencia sobre nosotros para hacer primar nuestra autonomía es doblemente imposible. Lo es, en primera instancia, porque para que nuestra autonomía sea tal, debe, en gran medida, ser si bien construida por uno mismo, también reconocida por los otros. ${ }^{2}$ En este sentido, debe insertarse en el entorno en que vivimos y ser reconocida por quienes nos rodean; sin su debido reconocimiento carecerá de la fuerza necesaria para ser, de hecho, operante.

En segunda instancia porque, ontológicamente, la libertad se ejecuta dentro de un marco de posibilidades dadas; es decir, se encuadra en un horizonte de pensamiento cultural y epistémico en el que los otros y lo otro constituyen elementos ineludibles. No se puede pensar si quiera obrar de modo tal que no se conozca. Para optar se requiere optar sobre algo y ese algo es, de suyo, limitado y concreto; luego, no puede ser absoluto, y optar por él sería renunciar a algo que no es él; así, elegir implica dejar de lado otras opciones distintas a lo que se ha elegido.

La diferenciación histórica entre sexo y género, más obediente al decaimiento de la soberanía individual que a su encumbramiento, resalta que la capacidad para decidir aún por sobre la naturaleza es absoluta en el ser humano; por ende, lo que él puede construir con o sin el reconocimiento de la biología de su propia vida, es lo 
único importante y verdadero. Está claro entonces que este desfase implica necesariamente no tanto un desplazamiento de lo verdadero a lo falso, como un ocultamiento de lo verdadero en donde recae la necesidad de concebir una libertad enraizada en la propia ontología y en la propia realidad del mundo y sus exigencias.

El no reconocer que la libertad implica, a su vez, una renuncia, ofusca el entendimiento del compromiso con la cosa elegida y, a su vez, desplaza también la actitud ética del cuidado que es, como veremos más adelante, la esencia del giro ético en las corrientes actuales.

\section{Vulnerabilidad}

Hablar de vulnerabilidad es apelar a la esencia misma del ser humano; es, por ende y ante cualquier afirmación posterior, una condición antropológica del ser humano.

El ser humano lleva, pues, en sí mismo, el sello indeleble de su vulnerabilidad; es decir, al ser una substancia finita, muere $y$, como dice Pascal, no sólo muere sino sabe que muere (Pascal, 2003). Así, su vida se convierte en una lucha por su sobrevivencia, pues su existencia es tan breve que puede ser amainada en cualquier momento y bajo cualquier circunstancia. La enfermedad, el dolor, el sufrimiento, la experiencia de la propia contradicción humana y de la vida como proyecto, como algo que se debe hacer porque no está previamente, constituyen elementos amalgamantes de su propia vulnerabilidad.

Por ello, hay que hablar, en un primer momento de la vulnerabilidad antropológica para, en un segundo momento, conectarla con la vulnerabilidad social de donde emana la reflexión bioética.

Hablar de vulnerabilidad es apelar a la esencia misma del ser humano; es, por ende y ante cualquier afirmación posterior, una condición antropológica del ser humano. 
Para Francisco J. De la Torre, existen cuatro columnas de la vulnerabilidad: la fragilidad, la desnudez, la posibilidad de desprecio y la debilidad (De la Torre, 2017, p. 155).

La fragilidad se refiere al daño que se puede hacer y que podemos sufrir en función de nuestra condición espacio-temporal, como afirma Feito: «el ser humano, que puede ser herido por ser frágil, y que queda no sólo lastimado, sino también marcado por la cicatriz, recuerdo del dolor, es víctima de su propia condición mortal» (Feito, 2007, p. 9).

Por su parte, la desnudez es el estado de total indefensión del ser humano pero, al tiempo, es el estado de mayor solicitud de responsabilidad ética, ya que impide quedar indiferente ante el rostro sufriente del otro, pues su desnudez es signo de su autenticidad. ${ }^{3}$ (Levinas, 1974, pp. 55-56).

Al respecto, la tercera característica de la vulnerabilidad humana, la posibilidad de desprecio, apela necesariamente al reconocimiento de uno mismo; es decir, a la posibilidad del encuentro con aquel que se presenta como frágil y vulnerable y que asemeja las características propias de fragilidad y vulnerabilidad y, por ello, se convierte en espejo de uno mismo (Taylor, 2001, p. 55).

Por último, la debilidad que nos coloca en situación de «caer», de ser vencidos, heridos, lastimados, de fracasar, de no cumplir, de cometer errores e incluso, de lastimar también a otros, descansa en lo que Ricoeur ha llamado labilidad, y que se asienta sobre el ideal de infinitud y la realidad finita que somos; este anhelo no cumplido le provoca tristeza y frustración al ser humano por no poder alcanzar lo que desea (Calvo, 1991, p. 97).

Estas cualidades representan el concepto de vulnerabilidad antropológica, que admite que todos somos vulnerables en función de nuestra humanidad y de nuestra posibilidad de ser heridos o lastimados ya sea corporalmente, moral, psicológica o integralmente.

Hay que recordar que la condición ontológica de la persona va de la mano con la acción, remarcando que la dignidad humana se refleja en las acciones humanas (no queda reducida a éstas pero sí 
se refleja en las mismas por el propio dinamismo humano de la acción), por ende, en la construcción y conquista de la libertad. Así, la libertad no es ya sólo una cualidad, sino una capacidad que, para ejercerse requiere, por su parte, del agenciamiento necesario.

Es darse cuenta de que nuestra vida es un proyecto, y esto nos coloca en una constante de vulnerabilidad, pues puede sobrevenir la debilidad, la desnudez, la fragilidad que amenazan este proyecto de vida. Así, surge la paradoja del ser humano en cuanto a que se sabe digno y libre pero a la vez vulnerable y amenazado por su entorno y por los otros. Así, Feito afirma: «La vulnerabilidad antropológica intrínseca es, entonces, no sólo una afirmación de nuestra impotencia o debilidad, sino, antes bien, una constatación de la vida como quehacer, como algo por construir, desde nuestra radical finitud» (Feito, 2007, p. 10).

En resumen, la vulnerabilidad antropológica implica, por un lado, la misma condición frágil y mortal del ser humano pero, a la vez, la relación con los otros y con su entorno, la capacidad para ir ejerciendo su libertad y forjando su identidad, por ello, surge la vulnerabilidad social.

La vulnerabilidad social proviene del ser esencialmente relacional de ser humano como afirmaba Aristóteles (Aristóteles, 2011, Libro II, $1253^{\mathrm{a}}$ ).

Como se afirmó antes, estar en el mundo implica ya un habérnoslas con el mundo; es decir, necesariamente soy y actúo con lo otro y con otros, y en este impacto recibido de los otros quedo influenciado por ellos y por el entorno. De esta manera hay condiciones o circunstancias que me colocan en un mayor riesgo de quedar afectado por ellas y si a éstas se le suma ya de por sí vulnerable condición antropológica, entonces el riesgo es doble y la afectación superior.

El entorno y el ambiente, la situación histórica, el poder adquisitivo de las personas, las leyes del mercado, la cultura y composición geográfica de un lugar, el clima, la disponibilidad y accesibilidad a los recursos y hasta los prejuicios y creencias predominan- 
tes constituyen factores que pueden vulnerar a las personas. A esta doble posibilidad de ser herido o lastimado algunos le llaman «espacios de vulnerabilidad»(Feito, 2007, p. 11), siendo condiciones que exponen a la persona no sólo a un mayor riesgo, sino que la colocan en un grado de mayor dificultad para defenderse o valerse por sí mismas.

Preciso es señalar aquí que se puede ir aumentando el grado de vulnerabilidad de una persona en cuanto a que, si bien existen riesgos sobre los cuales no se tienen el control humano y que lastiman a la persona, si ésta, históricamente, ha carecido de los medios para sobreponerse a dichas condiciones adversas, su vulnerabilidad aumentará, llegando incluso a presentar una triple dimensión: la vulnerabilidad antropológica (propia de todo ser humano); la vulnerabilidad social provocada por situaciones externas que se sitúan fuera del control humano, y la vulnerabilidad social producida por la falta o carencia de recursos (físicos y materiales, psicológicos e incluso jurídicos) para sobreponerse al riesgo.

De esta manera se puede clasificar la vulnerabilidad social en externa, que son los factores detonantes de la situación de crisis, algunos de ellos que se encuentran fuera de control como los desastres naturales y otros que recaen sobre el control del ser humano -y que son los que posteriormente nos interesarán más por su carácter reversible- como la marginación y exclusión social, los genocidios o los flujos migratorios por situaciones de guerra o desplazamientos forzados. Pero también se encuentran los factores internos, que versan sobre la capacidad para sobreponerse a los factores externos, y éstos tienen que ver con las condiciones de justicia social de cada grupo humano. De aquí que haya grupos humanos que, por haber carecido históricamente de reconocimiento moral o jurídico, quedan más expuestos y vulnerables para defenderse o salir adelante de situaciones adversas. En estos, el nivel de vulnerabilidad es mucho mayor. Esto ocurre, como veremos a continuación, en los grupos que han adoptado la ideología de género como constitutivo de su ser y de su actuar. 


\section{La ideología de género como vulnerabilidad social: el análisis desde la Bioética}

En el primer apartado de este capítulo se abordó el origen de la ideología de género como residuo de la discusión filosófica desprendida de las nociones de «género» $\mathrm{y}$ «sexo», pero alimentada por las categorías de «cultura» y «naturaleza». En este momento daremos algunas pautas que quieren argumentar cómo la ideología de género ha sido causante de una mayor exposición a la vulnerabilidad de ciertos grupos humanos como el colectivo LGTBI+.

En primer lugar, al separar la base biológica de la sexualidad de la integridad de la persona como unidad, la ideología de género ${ }^{4}$ marcó una libertad absoluta para la persona, tan absoluta que queda expuesta a deseos e inclinaciones tan pasajeras como pasajeras sean las emociones que experimentamos diariamente los seres humanos.

Así, situar a la persona a la total carencia de fundamentos y ubicarla como un ser en constante construcción, pero sin arraigos ni biológicos ni identitarios, permite una concepción des-dignificada de la misma; esto es, la coloca en el plano del resto de los entes que no tienen una necesidad de desenvolverse en el mundo y habérselas con la realidad. Lo coloca como una realidad cerrada y volcada hacia sí mismo pero, también por ende, expuesta a lo que los otros quieran hacer con ella.

Los entes materiales se convierten en utensilios en la medida en que es el ser humano quien les confiere el sentido de su existencia, pero no porque ellos tengan un sentido o, menos aún, una vocación de ser, sino porque están para el ser humano y, a diferencia de éste, su esencia es una clausurada y sujeta a lo que otros decidan de ella.

En cambio, el ser humano es un ser de realidades (López Quintás, 2000, p. 36), que construye su mundo y se construye en sus acciones; es un ser que participa en y con los demás en la construcción de su propio sentido y del sentido de ser de los demás 
(Gevaert, 2001, p. 14). Por esto no podemos decidir por otro, porque cada uno es una unidad sustancial y capacidad de razonamiento propio y, por lo tanto, de elecciones propias. Nuestra esencia no es una ya dada y prefijada por otro, sino que es un misterio (Gevaert, 2001, p. 14), y permanece en continuo cambio y reificación en la medida en que vamos siendo afectados por los otros; no obstante, este devenir tampoco es infinitamente abierto, sino que recae siempre en esa substancia individual que somos cada uno y sobre la cual vamos aceptando ser modificados por el entorno o no. Siempre en referencia a lo que cada uno es, incluso antes de interactuar con el mundo.

Ahora bien, si se afirma que la persona no tiene una tarea que hacer mediante su libertad y su juicio, entonces se pierde el sentido y el horizonte de realidad sobre el cual descansan nuestras decisiones. Perdido este horizonte, la persona asiste al desmoronamiento de su valor intrínseco, rechazando con ello cualquier posibilidad de sustento. Queda, así, reducida su existencia a la mera existencia, al ámbito de los seres que no tienen libertad y que tampoco necesitan tenerla.

Al proponer esta carencia de fundamentos ontológicos que permitan el intercambio de contenidos sin alterar una esencia propia de cada persona, y al no tener que formar su ser ni forjar su identidad en base a algo, la ideología de género permea en las estructuras más íntimas de las personas, otorgándoles una supuesta libertad para elegir su género y actuar en consecuencia, dejando expuestas a las personas a factores sociales de reconocimiento que no siempre terminan por ser tal. De esta manera, aumenta el grado de vulnerabilidad, pues las personas no cuentan, en su mayoría, con los mecanismos que los provean de la capacidad para su propia defensa.

Lo anterior queda evidenciado con los colectivos LGTBI+, en donde se ha establecido una lucha frontal por la defensa y reconocimiento de sus derechos pero, al mismo tiempo, han sido grupos sistemáticamente no reconocidos y vulnerados, incluso, pareciera que, entre mayor sea su causa, más tipos de violencia ${ }^{5}$ los aquejan y 
esto en función de que exigen una actitud por el hecho de ser LGTBI+, pero no por el hecho de ser personas, y al momento de particularizar los derechos, los mecanismos para defenderlos dejan de ser accesibles a todos también, y se adecuan a grupos particulares generando una mayor deficiencia a su accesibilidad.

Las personas LGTBI+ no tienen derechos por ser tales, sino por el hecho de que son personas. Ahora bien, recordando lo que se dijo acerca de las tres dimensiones de la vulnerabilidad, una persona LGTBI+ es una persona vulnerable, en primera instancia por ser persona y por su condición humana; en segundo lugar, por los factores sociales que exponencian su vulnerabilidad, ya sea por las circunstancias de vida de cada uno o bien por los prejuicios sociales existentes alrededor de quien se declara homosexual, bisexual, transexual, etc. El ámbito de ocultamiento y clandestinidad fomenta sus conductas de riesgo y alberga una mayor vulnerabilidad para ellos. En tercer lugar, en múltiples ocasiones, por la segunda vulnerabilidad explicada, sufren vejaciones y menosprecios, malos tratos y exclusión social, y carecen de los medios para denunciar o defenderse ya que, también por los prejuicios y creencias existentes, denunciar los fuerza a salir de la clandestinidad y esto, es ya también asumir un riesgo mayor.

Por lo anterior, el reclamo de sus derechos y, más aún, de derechos específicos para ellos, se centra en solicitar un reconocimiento que les provea de los medios necesarios para salir del ocultamiento sin riesgos y/o amenazas.

$\mathrm{Si}$ bien es cierto que ni todos los colectivos LGTBI+ proclaman y defienden la ideología de género, lo es también que ésta no fortalece sino que radicaliza sus peticiones.

La ideología de género, al rechazar el fundamento ontológico de la persona como mujer o varón, ha proclamado, a su vez, muchas «ontologías», que hacen creer que es necesario acentuar muchos reconocimientos dejando su adecuado desarrollo a la medida en que estos reconocimientos se den de hecho, pero esto es hacer depen- 
der la dignidad como valor intrínseco de toda persona de condiciones regulatorias, es decir, de acciones negativas.

Con lo anterior, el sujeto queda en un estado de mayor vulnerabilidad ya que, de no reconocerse su condición, su «ontología» queda insatisfecha y más fácilmente dañada.

La recuperación, por ello, del sustrato ontológico de la persona, unido a su reconocimiento pleno, afirmaría no muchas sino una ontología, y no dependiente de factores sociales. La ideología de género, en un afán por defender una autonomía y dominio de la naturaleza, expone más a la persona a ámbitos de realidad que hacen más grande aún su ya de por sí marcada vulnerabilidad.

De esta manera, las acciones afirmativas que no sólo reparen el daño del no reconocimiento, sino que doten a la persona de las herramientas para defenderse y sobreponerse implica, forzosamente, reconocer la dignidad de cada persona en cuanto tal, no en cuanto sus categorizaciones. Así, se debe reconocer al que es homosexual no porque es homosexual sino porque es persona; el camino debe ir por el lado del retorno a la persona y la asunción de un sustrato ontológico en cada uno.

Muchas han sido las consecuencias de la ideología de género en temas de Bioética, principalmente por las prácticas en donde se expone a la persona a mayores grados de vulnerabilidad, por implicar procedimientos invasivos y que comportan altos riesgos para la vida y salud de la persona

Un ejemplo de cómo la ideología de género expone a la persona a una mayor vulnerabilidad son las intervenciones quirúrgicas de reasignación sexo-genérica, a partir de las cuales es posible debatir el principio terapéutico.

Desde la bioética, se considera el principio terapéutico como aquel que permite una acción a intervención sobre una parte del cuerpo de la persona con el fin de preservar el todo y siempre y cuando exista una condición necesaria para intervenir. Se habla de necesidad médica cuando, en caso, de no intervenir, la vida de la persona corre peligro inminente y ésta puede, incluso, morir. Así, 
el principio terapéutico implica asumir los riesgos de una cirugía o de un procedimiento en beneficio del todo; el bien mayor prima en la decisión.

Ahora bien, las cirugías de reasignación de sexo o de concordancia sexo-genéricas, solicitadas y permeadas por la creciente ideología de género, tienen por fundamento hacer «concordar» el sexo -biológico- de la persona con el género que ha asumido la misma. Chárries Cordero las define como:

«La cirugía de reasignación sexual (CRS) consiste en procesos quirúrgicos que las mujeres y los hombres transexuales llevan a cabo para armonizar su sexo anatómico con su identidad sexual. Puede centrarse en los genitales, denominada cirugía de reconstrucción genital, y en la que se pueden distinguir operaciones como la vaginoplastia, la metadoioplastia o la faloplastia. Pero también existen operaciones feminizantes o masculinizantes de caracteres sexuales no genitales, como puede ser una cirugía facial o una mastectomía» (Chárries, 2013, p. 24).

Así, salvo en los casos en que se haya diagnosticado una necesidad apremiante y urgente en donde se considere que la vida de la persona corre peligro, deberá procederse a dichas cirugías, ya que los riesgos de las mismas sobrepasan los beneficios obtenidos.

El simple hecho de presentar una identidad sexual diferente de la orientada naturalmente por el sexo biológico puede obedecer más a modas y discursos sociales normativos en la época histórica que a verdaderas causas de trastornos psicológicos que puedan poner en riesgo la salud física o mental de la persona.

Cabe mencionar que sí existe el trastorno de identidad de género o disforia de género ${ }^{6}$ y que sí se presenta con relativa frecuencia entre la población; incluso se han establecido ya criterios de inclusión de paciente en las cirugías de cambio de sexo (Pavon, 2000, p. 783), ya que se considera que este trastorno si afecta la salud mental de las personas, mas no debe confundirse con cualquier petición que así lo expresara. 
Aunado al principio terapéutico, debe valorarse el principio de integridad, que implicaría una exigencia por respetar el lugar de manifestación de la persona que es su cuerpo y salvaguardar sus valores y preferencias en concordancia con esto; así, al intervenir sobre el cuerpo de una persona, se está interviniendo sobre su integridad en cuanto tal y, de no asumir esta posición, ésta podría quedar dañada irreversiblemente.

Algunas otras formas de vulnerabilidad a las que quedan sometidas las personas al aceptar la separación sexo-género en el ámbito de la salud incluyen: faltas al debido consentimiento informado, asumiendo que son inferiores o incapaces para tomar decisiones, malos tratos y/o burlas por parte del personal médico, prácticas de esterilización forzada, tratamientos hormonales no consentidos, etcétera. $^{7}$

Un enfoque bioético necesitaría, además de revisar las condiciones del principio terapéutico, analizar los temas relativos al consentimiento informado que resulta ser un ámbito que expone a los pacientes a una mayor vulnerabilidad, al no darles acceso a información debida y veraz sobre su condición y los posibles tratamientos existentes; hay que recordar que el consentimiento informado debe proporcionarse, por ley, en todos los procedimientos invasivos, que comporten riesgos para la salud y en temas de planificación familiar. El no dar información o darla de forma fragmentada es incurrir en una falta ética grave, pues en el tema que nos compete, el informar sobre las diferencias entre sexo y género pero también sobre la continuidad de estos conceptos implica asumir la condición ontológica de la persona como un ser sexuado y su manifestación como hombre o como mujer (Burgos, 2011, p. 9), sin que necesariamente tengan que contravenirse estas nociones.

Recordemos aquí que la ideología de género no es la teoría de género como se explicó al inicio de este capítulo y que, por el contrario, resulta más un residuo que se sitúa en la radicalización de la separación natural y social que en la fundamentación teórica de las nociones, así que, de alguna manera, este entendimiento contribuye 
a la no información y, en temas de salud, a la falta de consentimiento informado en la relación profesional de la salud-paciente colocándolo en un mayor grado de vulnerabilidad.

\section{Conclusiones}

La bioética tiene un papel trascendental especialmente en lo referente a los grupos vulnerables y a las personas vulneradas, pues deberá marcar las pautas y acciones necesarias para intentar, primero recobrar y nivelar las condiciones que inicialmente pusieron a esa persona en mayor exposición y, en segundo lugar, asegurar que esa persona cuente con los mecanismos que le permitan afrontar su vida y superar los obstáculos que ésta le presente.

Pasar de la responsabilidad a la solidaridad resulta imprescindible si buscamos que la bioética atienda y mejore condiciones injustas y desiguales. La atención justa y solícita del frágil y necesitado constituye un grito urgente para la ética y para la bioética; más aún, cuando existen ideologías que siguen insistiendo en dejar al ser humano desprovisto de un suelo firme e inalterable, y sujeto a las variables del entorno social en donde prevalecen los prejuicios y las ideas marginales.

Resulta, por todo lo anterior, imperante atender el dualismo sexo-genérico desde una mirada bioética compasiva que corriga pero abrace, que sea capaz de develar la verdad del ser humano a la luz de la empatía y del acompañamiento solidario, no en virtud del cuidado necesario, sino en virtud del reconocimiento de nuestra común condición humana vulnerable.

\section{Notas bibliográficas}

${ }^{1}$ Dependiendo del tipo de diversidad sexual que se defienda, se admitirá o no un sustento biológico o un componente fijo e inamovible para la práctica de las conductas que de éste derivan. 
2 Sobre la teoría del reconocimiento hablaré más adelante como un constitutivo de nuestra identidad.

${ }^{3}$ Levinas habla de la huella que deja el rostro del otro. Levinas, E. (1974). El humanismo del otro hombre. Madrid, Siglo Veintiuno, 1974, pp. 55-56.

${ }^{4}$ Resalto aquí que me refiero a la «ideología» y no a la «teoría», ya que, como explicamos al inicio, la teoría de género tiene bondades intrínsecas al haber detectado los roles socialmente construidos que carecen de fundamentación ontológica; en este sentido, la ideología se toma como la radicalización de la teoría.

${ }^{5}$ Para una mayor referencia, ver el informe Comisión Interamericana de Derechos Humanos (2015). Violencia contra las personas lesbianas, gay, bisexuales, trans e intersex en América. OAS. Documentos oficiales. Ser. I. OEA.

${ }^{6}$ Agregado al DSM-V en 2013. Para una mayor referencia respecto de los cambios del DSM-IV al DSM-V ver el sitio: https://www.psychiatry.org/psychiatrists/ practice/dsm/proposed-changes. Fecha de última consulta: 7 de septiembre del 2019.

${ }^{7}$ Resulta interesante leer los distintos tipos de violencia terapéutica reportada. Cfr. Comisión Interamericana de Derechos Humanos. (2015). Op. Cit. Sección "formas y contextos de la violencia».

\section{Sitios electrónicos consultados}

WHO. «What do we mean by sex and gender». En Gender, women and health. Disponible en: http://www.who.int/gender-equity-rights/understanding/gender-definition/en/Fecha de última consulta: 2 de septiembre de 2019.

\section{Referencias bibliograficas}

Aristóteles. Política. Espasa Calpe, Madrid, 2011.

BeAuvoir, Simone. El segundo sexo. Cátedra, Madrid, 2005.

BONDER, GLORIA. «Género y subjetividad. Avatares de una relación no evidente». En Género y Epistemología. Mujeres y disciplinas. Programa interdisciplinario de estudios de género. Universidad de Chile, Chile, 1998. Disponible en http:// www.enlinea.cij.gob.mx/Cursos/Hospitalizacion/pdf/bonder.pdf Fecha de última consulta: 2 de septiembre de 2019.

BURgos, JUAN MANUEL. «Dos Formas de afrontar la identidad sexual: personalismo e ideología de género». En Aparisi, A. Persona y género. Thomson ReutersAranzadi, Pamplona, 2011.

Calvo, Tomas. Paul Ricoeur. Los caminos de la interpretación. Anthropos, BarceIona, 1991.

ChARRIES CORDERO, MAYRA. "La transexualidad: ¿construcción de una identidad?». En Revista Griot. 2013; 6(1). 
Ma. E. de los Ríos Uriarte

COMISIÓN INTERAMERICANA DE DERECHOS HumANOS. Violencia contra las personas lesbianas, gay, bisexuales, trans e intersex en América. OAS. Documentos oficiales. Ser. I. OEA. 2015.

DE LA TORRE, FRANCISCO JAVIER. «Vulnerabilidad. La profundidad de un principio en bioética». En Perspectiva teológica. Enero-Abril 2017; 49(1): pp. 155-176.

FEITO, LYDIA. «Vulnerabilidad». En Anales del sistema sanitario de Navarra. 2007; 30, Suplemento 3, pp. 7-22.

FoCuAULt, Michel. Historia de la sexualidad I: la voluntad de saber. Trad. Ulises Guiñalú. 25a ed. Siglo XXI Editores, México, 1998.

GeVAERT, JosePh. El problema del hombre. Sígueme, Salamanca, 2001.

LEVINAS, EMMANUEL. El humanismo del otro hombre. Siglo XXI Editores, Madrid, 1974.

LOCKE, JoHn. Ensayo sobre el gobierno civil. $3^{a}$ ed. Porrúa, México, 2003.

LÓPEZ QUINTÁS, ALFONSO. Inteligencia creativa. BAC, Madrid, 2000.

Nozick, Robert. Anarquía, Estado y Utopía. Trad. Tamayo, R. Fondo de Cultura Económica, México, 1998.

Pascal, Blas. Pensamientos. Losada, Buenos Aires, 2003.

PAVÓN DE LA PAZ, ISABEL. "Tratamiento de reasignación de sexo en pacientes transexuales». En Med. Clin. Barcelona. 2000; 115: pp. 783-788.

TAYLOR, CHARLES. El multculturalismo y la política del reconocimiento. Fondo de Cultura Económica, México, 2001. 